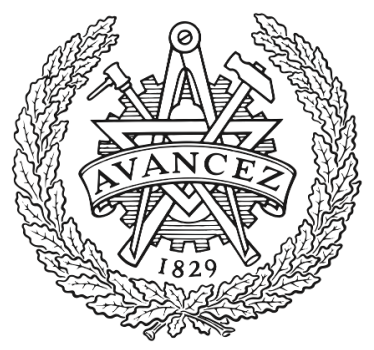

CHALMERS

UNIVERSITY OF TECHNOLOGY

\title{
Comparison of Automotive FMCW and OFDM Radar Under Interference
}

Downloaded from: https://research.chalmers.se, 2023-04-26 12:32 UTC

Citation for the original published paper (version of record):

Carvajal, G., Keskin, F., Aydogdu, C. et al (2020). Comparison of Automotive FMCW and OFDM Radar Under Interference. IEEE National Radar Conference - Proceedings, 2020-September. http://dx.doi.org/10.1109/RadarConf2043947.2020.9266449

N.B. When citing this work, cite the original published paper. 


\title{
Comparison of Automotive FMCW and OFDM Radar Under Interference
}

\author{
Gisela K. Carvajal*, Musa Furkan Keskin ${ }^{\dagger}$, Canan Aydogdu ${ }^{\dagger}$, Olof Eriksson $^{\ddagger}$, Hans Herbertsson ${ }^{\ddagger}$, \\ Hans Hellsten $^{\S * *}$, Emil Nilsson**, Mats Rydström ${ }^{\ddagger}$, Karl Vänas ${ }^{\|}$, and Henk Wymeersch ${ }^{\dagger}$ \\ *Qamcom Research \& Technology, Gothenburg, Sweden \\ ${ }^{\dagger}$ Chalmers University of Technology, Gothenburg, Sweden \\ $\ddagger$ Veoneer, Vårgårda, Sweden; ${ }^{\S}$ Saab, Linköping, Sweden \\ ${ }^{* *}$ Högskolan i Halmstad, Halmstad, Sweden; "Volvo Cars, Gothenburg, Sweden \\ Email: gisela.carvajal@qamcom.se
}

\begin{abstract}
Automotive radars are subject to interference in spectrally congested environments. To mitigate this interference, various waveforms have been proposed. We compare two waveforms (FMCW and OFDM) in terms of their radar performance and robustness to interference, under similar parameter settings. Our results indicate that under proper windowing both waveforms can achieve similar performance, but OFDM is more sensitive to interference.
\end{abstract}

\section{INTRODUCTION}

There have been many studies about the implementation of different types of radar for automotive applications [1]-[4]. Commonly used radar waveforms are the Frequency Modulated Continuous Wave (FMCW) and Orthogonal Frequency Division Multiplexing (OFDM). The efficient deployment of joint radar and communication systems have attracted a lot of attention in recent years (see [5] for a recent review). Both FMCW and OFDM radars are susceptible to interference. As the number of radar transceivers operating throughout the traffic environment is foreseen to increase rapidly over the coming years, radar interference is also expected to increase [1]. This motivated a number of studies to investigate radar interference mitigation techniques (see for example [1], [6][8]). In [3], it is shown that the interference among FMCW radars may result in ghost target detection, providing an analytical calculation for the probability of such event. Co-channel interference becomes more severe if radars share the frequency spectrum with communication systems [9], however with such systems, a central controller may manage resource allocation to avoid co-channel interference [1]. In [10], the effects of interference in FMCW and phase-modulated continuous-wave radars are calculated in terms of interference-to-noise ratio.

In applications such as autonomous driving, OFDM radar has a number of advantages over FMCW radar; it can offer high-rate data transmission through the dual-functionality of the OFDM waveform [11], it utilizes the same hardware as the communication unit, and it enables independent estimation of range and speed [12, Ch. 3]. In [13], a comparison between the OFDM and the chirp-sequence radars is presented, where

978-1-7281-8942-0/20/\$31.00 (C)2020 IEEE it is claimed that if the slope of the frequency ramps in the chirp sequence signal goes to infinity, the baseband signal for both technologies become the same and hence have the same performance; numerical examples for these results are provided by the same authors in [14].

In this paper, we present a comparison between OFDM and FMCW radar, with and without interference. Our specific contributions are: (i) we evaluate the performance of OFDM and FMCW radar under similar parameter settings in terms of range and velocity resolution and sidelobe levels; (ii) we evaluate the impact of interference on OFDM and FMCW radar detection.

\section{RADAR WAVEFORMS}

In the following, we consider FMCW and OFDM radar systems that work with similar parameters, including the same carrier frequency, bandwidth, transmitted power, and integration times. An overview of the FMCW and OFDM systems is depicted in Fig. 1, with details provided below.

\section{A. FMCW Radar}

We consider an FMCW waveform with the carrier frequency $f_{c}$, the chirp slope $\alpha$ and the number of chirps $M$ [1], [15]

$$
x(t)=\sum_{m=0}^{M-1} s(t-m T),
$$

where the individual chirps of duration $T$ are given by

$$
s(t)=e^{j 2 \pi\left(f_{c} t+0.5 \alpha t^{2}\right)} \operatorname{rect}_{T}(t)
$$

with $\operatorname{rect}_{T}(t)$ denoting a square pulse with amplitude 1 for $0 \leq t \leq T$ and 0 otherwise. Suppose there exists a single target at range $R$ and velocity $v$, with round-trip propagation delay $\tau=2 R / c$ and normalized Doppler shift $\nu=2 v / c$. Then, the received signal for the $m$ th chirp can be expressed as

$$
r_{m}(t)=\gamma s(t-m T-\tau+t \nu)+z_{m}(t),
$$

for $0 \leq t \leq M T$, where $\gamma$ is the radar channel gain covering the path loss and radar cross section effects, and $z_{m}(t)$ denotes the measurement noise. Note that time-dependent delay $\tau-$ $t \nu$ decreases over time for positive Doppler shifts $\nu$. After 

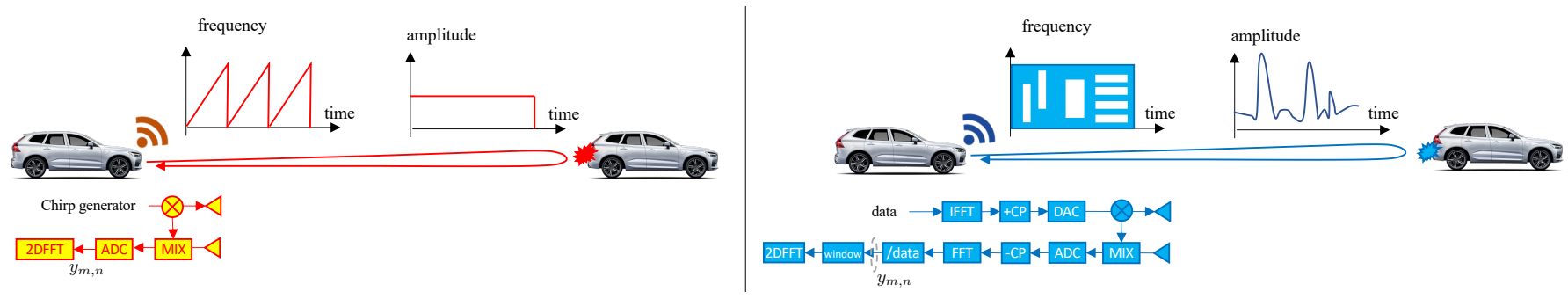

Fig. 1. Comparison between FMCW radar (left) and OFDM radar (right). OFDM involves a more complex transmit and receive signal processing chain, but can control the data, allowing more freedom in time and frequency. The high PAPR of OFDM is a limiting factor. FMCW relies on chirp signals with low PAPR, and simple transmit and receive signal processing.

dechirping $r_{m}(t)$ via conjugate mixing with the transmit signal $s(t)$, we obtain the discrete-time signal model as [1]

$$
y_{m, n}=\gamma e^{j 2 \pi\left(-\alpha \tau+f_{c} \nu\right) n T_{s}} e^{j 2 \pi f_{c} \nu m T}+z_{m, n},
$$

where $m$ and $n$ represent slow-time and fast-time indices, respectively. The range-Doppler coupling term $f_{c} \nu n T_{s}$ in (4) is typically negligible for practical automotive settings [16, Ch. 4.6.4]. From (4), delay-Doppler estimation can be performed by applying two-dimensional Fourier transform across slow-time and fast-time dimensions. The FMCW signal and processing chains are shown on the left side of Fig. 1.

\section{B. OFDM Radar}

In this type of radars, the waveform used is an OFDM signal known from communications. The processing details are documented in [12], [17], [18] and are summarized here. The transmit signal consists of parallel orthogonal subcarriers, each modulated with a data. The resulting baseband time-domain signal is expressed as [18]

$$
x(t)=\sum_{m=0}^{M-1} \sum_{n=0}^{N-1} D(m N+n) e^{j 2 \pi n \Delta_{f} t} \operatorname{rect}_{T}(t-m T),
$$

where $N$ represents the number of subcarriers, $M$ is the number of consecutive symbols evaluated, $\Delta_{f}$ is the subcarrier spacing, $T=T_{\mathrm{cp}}+T_{\mathrm{sym}}$ is the OFDM symbol duration consisting of the cyclic prefix $(\mathrm{CP})$ duration $T_{\mathrm{cp}}$ and the elementary symbol duration $T_{\mathrm{sym}}$, and $D(n)$ represents the complex modulation symbol (the arbitrary data modulated with a discrete phase modulation technique, e.g., quadrature amplitude modulation (QAM)).

The processing of the backscattered signal consists of the following steps [12, Ch. 3.2]: (i) removal of the $\mathrm{CP}^{1}$, (ii) Fourier transform over the elementary symbol duration, and (iii) element-wise complex division by the transmit symbols. Then, for the $n$th subcarrier of the $m$ th OFDM symbol, we obtain

$$
y_{m, n}=\gamma e^{-j 2 \pi n \Delta_{f} \tau} e^{j 2 \pi f_{c} \nu m T}+z_{m, n} .
$$

\footnotetext{
${ }^{1}$ The $\mathrm{CP}$ duration is assumed to be larger than the round-trip time of the furthermost target [12, Ch. 3.2.1]. In contrast to OFDM communications, OFDM radar is mono-static and does not require synchronization to detect the CP interval of the incoming signal.
}

Similar to FMCW processing, taking the two-dimensional Fourier transform over frequency and time dimensions in (6) yields estimates of range and Doppler parameters. The OFDM signal processing chain is shown on the right side of Fig. 1.

\section{QUALITATIVE COMPARISON}

In this section, we perform a comparison between FMCW and OFDM in terms of the properties and parameters. We consider the following aspects:

- Range resolution $(\Delta R)$ : for both OFDM and FMCW, we require that the total bandwidth $B>c /(2 \Delta R)$. Note that the instantaneous bandwidth, limited by the ADC sampling rate $B_{\text {adc }}$, may be much smaller. For OFDM, this can be realized through stepped-carrier approaches [19], while for FMCW this is realized inherently by the waveform.

- Velocity resolution $(\Delta v)$ : depends on the number of chirps for FMCW or the number of OFDM symbols for OFDM. Both are denoted by $M$, with $M>c /\left(2 f_{c} \Delta v T\right)$, where $f_{c}$ is the carrier frequency and $T$ is the chirp duration for FMCW or the symbol duration for OFDM.

- Maximum range $\left(R_{\max }\right)$ : For FMCW, this is determined by the ADC bandwidth $B_{\text {adc }}$ with $B_{\text {adc }}>2 R_{\max } \alpha / c$. For OFDM, the maximum range is limited by the cyclic prefix duration $T_{\mathrm{cp}}>2 R_{\max } / c$.

- Maximum velocity $\left(v_{\max }\right)$ : depends on the fast time duration, with $T \leq c /\left(2 f_{c} v_{\max }\right)$.

- Time-frequency utilization $U$ : for FMCW, $U=$ $M T B_{\text {adc }} \times u$, where $u \in(0,1]$ is the radar duty cycle. For OFDM, $U=M T B \times u \times f$, where $f \in(0,1]$ denotes the fraction of non-zero subcarriers during active transmission. This parameter is important for interference robustness as it determines the number of mutually noninterfering radars that can coexist.

- Peak-to-average power ratio (PAPR): is defined as PAPR $=\max \left[|x(t)|^{2}\right] / \operatorname{mean}\left[|x(t)|^{2}\right]$. For FMCW, PAPR $=1$. In OFDM, PAPR can be very high (e.g., a single OFDM symbol with unit mean power the PAPR can be as high as the number of sub-carriers $N$, when all sub-carriers transmit the same symbol). Practical OFDM systems scramble the information to be transmitted, which causes the transmit data to appear pseudo-random. In this case, it has been shown that PAPR rarely exceeds 
a value of $2 \log N$ [20]. A high PAPR is detrimental as it reduces the power efficiency of amplifiers.

\section{Simulation Results}

\section{A. Simulation Parameters}

In order to compare the detection of FMCW radar with OFDM radar it is necessary to set comparable parameters that would theoretically allow to have the received signal with the same resolution in range and velocity. In the following, we consider radar systems operating at $f_{c}$ of $77 \mathrm{GHz}$ with a bandwidth $B=B_{\text {adc }}$ of $200 \mathrm{MHz}$. The signal processing in the receiving stage is done with consecutive computation of FFTs in the range and Doppler domain. The range sampling is $N=1024$, while the Doppler sampling is $M=512$. This allows a range resolution $\Delta R=0.75 \mathrm{~m}$ and a velocity resolution $\Delta v=0.74 \mathrm{~m} / \mathrm{s}$. The antennas in both systems are set to transmit equal power $P_{t}=1 \mathrm{~W}$ with a field of view $20^{\circ}$ in azimuth and $10^{\circ}$ in elevation. The antennas are assumed to have a rectangular beam with gain $G=39 \mathrm{~dB}$. All elements, vehicle, target and interference are assumed to have a radar cross section $\sigma=10 \mathrm{dBsm}$. We assume a full bandwidth OFDM $(f=1)$ and i.i.d. 16-QAM data. Furthermore, the signal detection is affected by the application of windows in the time domain. We consider rectangular, Hamming and Dolph-Chebyshev ${ }^{2}$ windows [21], [22]. In order to focus on the signal behavior, noise sources are not considered and the detection is normalized.

\section{B. Performance without Interference}

Here we study the detection in range and velocity of a specific target. The aim of this is to compare the similarities and differences in detection performance of ideal FMCW and OFDM radar systems with different windows. For better observation we show the cuts of the range and velocity detection plots at the target location. In particular, here it is shown the detection of a target moving with a velocity of 30 $\mathrm{m} / \mathrm{s}$ when located at $120 \mathrm{~m}$ from the radar.

Fig. 2 shows the range detection cut for the target. As expected, the rectangular window is the one that produces highest sidelobes. Inherent to the waveform definitions, they result in different distributions of the received energy. In the case of FMCW radars, there is a coupling between range and Doppler estimation and the received echo has a high sidelobe level. We observe a sidelobe suppression after applying Hamming and Chebyshev windows in direct accordance to the window representation in the frequency domain. For OFDM, there is no such coupling and the OFDM signal has a maximum autocorrelation for only one position, which produces a low sidelobe level. The behavior of the OFDM signal results in a side-lobe attenuation over the whole FFT sample range when applying windowing. Fig. 3 shows the speed detection cut for the target with our FMCW and OFDM systems. The effect of the windows is analogous to the case of the range detection. However, one can observe slow variation in the signal for both

\footnotetext{
${ }^{2}$ The Dolph-Chebyshev window was set to $60 \mathrm{~dB}$ sidelobe suppression.
}

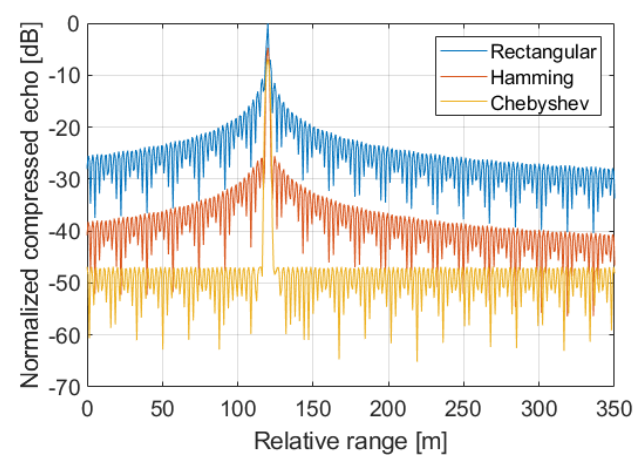

(a)

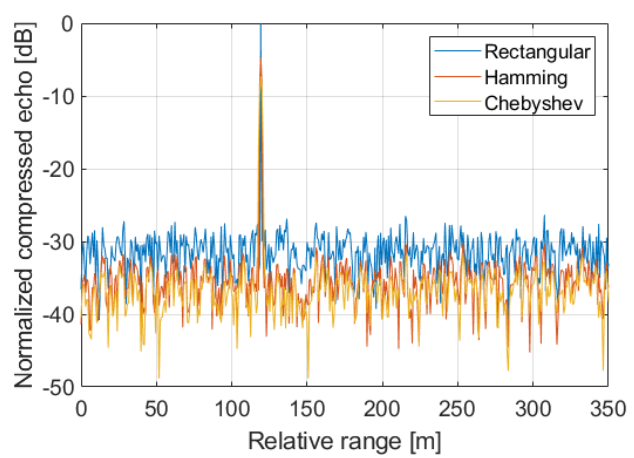

(b)

Fig. 2. Range profiles for a target at $120 \mathrm{~m}$ moving with $30 \mathrm{~m} / \mathrm{s}$ towards the radar. (a) and (b) show FMCW and OFDM detection respectively. The detection is affected by three window types: Rectangular, Hamming and Chebyshev.

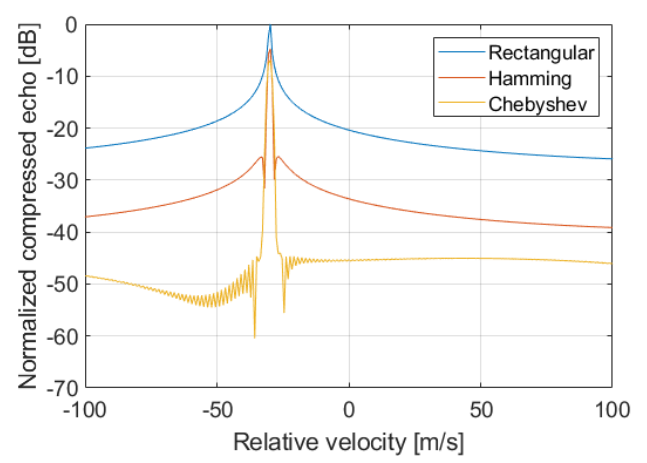

(a)

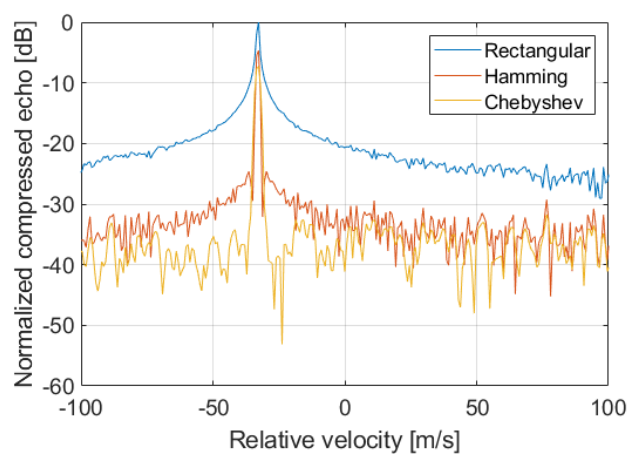

(b)

Fig. 3. Velocity profiles for the same target of Fig. 2. (a) and (b) show FMCW and OFDM detection respectively. 


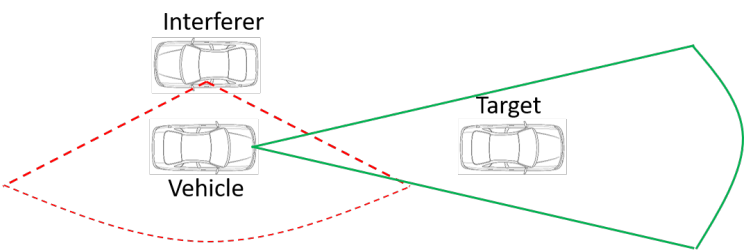

Fig. 4. Interference traffic scenario. Radar of interest has position $[0 \mathrm{~m}, 0 \mathrm{~m}]$ and velocity $30 \mathrm{~m} / \mathrm{s}$. The interferer has position $[0 \mathrm{~m}, 75 \mathrm{~m}]$ and velocity $0 \mathrm{~m} / \mathrm{s}$. The target has position $[75 \mathrm{~m}, 0 \mathrm{~m}]$ and velocity $30 \mathrm{~m} / \mathrm{s}$.

FMCW and OFDM due to the fact that the phase of the signal is assumed to be constant.

\section{Performance with Interference}

Interference signals can be generated in many ways by other sensors operating in the same frequency as the radar of interest and can even be produced by multipath backscatter of the radar signal or by leakage of the transmitted signal in the receiver circuit [7], [23]. It is also possible to have the case in which the target vehicle includes a radar that can be the source of interference. To simplify the analysis, here we consider a scenario where the target vehicle does not include a radar and where the source of interference is not in the field of view of the radar of interest. Here the power transmitted by both the studied radar and the interference is equal and assumed $1 \mathrm{~W}$. We also use a Chebyshev window in the range and Doppler signal processing. This scenario is shown in Fig. 4.

1) $F M C W$ : Fig. 5 compares the response pattern with different interference durations (long duration in Fig. 5a and short duration in Fig. 5b). In Fig. 5a the interference is coherent with respect to the radar signal. This leads to two identifiable "targets" (labeled A (interference) and B (target of interest)). The strongest detection is due to the interference signal in A, since although it is located at the same distance of $75 \mathrm{~m}$ of the target with respect to the radar, it has only oneway propagation loss. The velocity of $\mathrm{A}$ is $-30 \mathrm{~m} / \mathrm{s}$ since the detected Doppler for a velocity of $0 \mathrm{~m} / \mathrm{s}$ is subtracted from the radar vehicle velocity of $30 \mathrm{~m} / \mathrm{s}$. The target of interest is at the location B at a range of $75 \mathrm{~m}$ and a speed of $30 \mathrm{~m} / \mathrm{s}$. Here we notice that a very strong "target" A due to the interference. This strong interference can lead to saturation in the receiver that may impair the detection of the target of interest $\mathrm{B}$. When the interference signal is of a shorter duration, the response pattern in Fig. 5b shows that interference signal A is spread along the overall range at a velocity of $-30 \mathrm{~m} / \mathrm{s}$. In this case, the interference is no longer fully coherent with the signal of interest and the target B is now the strongest signal. Comparing this case with the coherent interference of Fig. 5, one could see that the noise floor level increases for the shorter interference pulse. This is consistent with observations in the literature as in [1], [3], [23]. These results exemplify that interference, varies greatly according to the scenario. The interference signal can appear as ghost targets or as an increased noise level, reducing the desired SNR of the receiving radar. For example, in the case that the target of interest would contain an interference

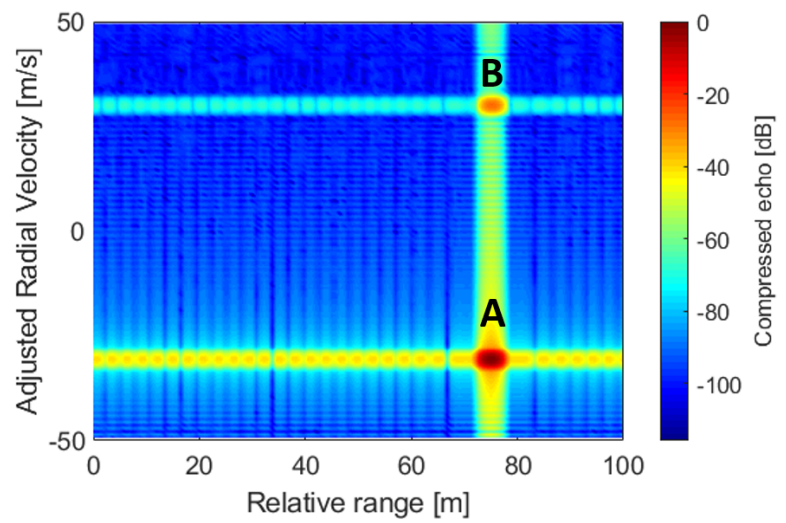

(a)

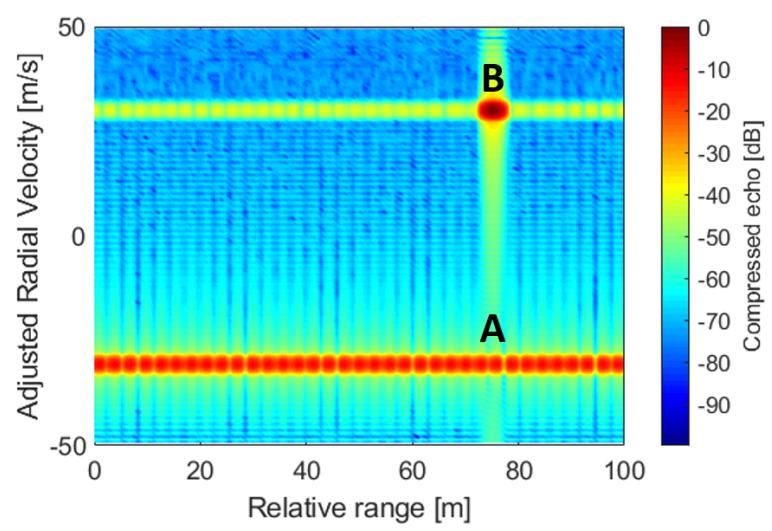

(b)

Fig. 5. Response pattern of signal detection for a vehicle equipped with an FMCW radar with waveform duration $T=5.12 \mu$ s in the presence of an interfering FMCW radar. The duration of the interference pulse is $5.12 \mu \mathrm{s}$ in (a), and $3 \mu$ s in (b).

radar, the target detection would be directly impaired by the interference and it may be not be discernible.

2) OFDM: Fig. 6 shows the response pattern for fullband OFDM radar with interference, according to the scenario of Fig. 4. Since the interference has a one-way propagation signal and overlaps with the signal of interest over the whole bandwidth and channel time the resulting detection shown in Fig. 6a makes it impossible to identify the compressed echo signal corresponding to the target of interest. Equivalent results have been shown in [7], which proposed to mitigate interference using an OFDM joint radar and communication system. Since the radar is capable of demodulating the interfering communication signals, these can then be subtracted from the reflected radar signal to improve its dynamic range. However, this method requires the a-priory knowledge of a pilot symbol in the interference signal. Similar to the FMCW study, we investigate the impact of less coherence in the interference for OFDM. In particular, when only 104 adjacent carriers of the interfering signal overlap with the total of 1024 carriers in the transmitted signal, Fig. $6 \mathrm{~b}$ indicates that target detection becomes possible (a zoomed-in version is shown in Fig. 6c). This would imply a limit for a channel overlapping of about 
$10 \%$.

\section{CONCLUSION}

We have compared FMCW and OFDM radar systems,

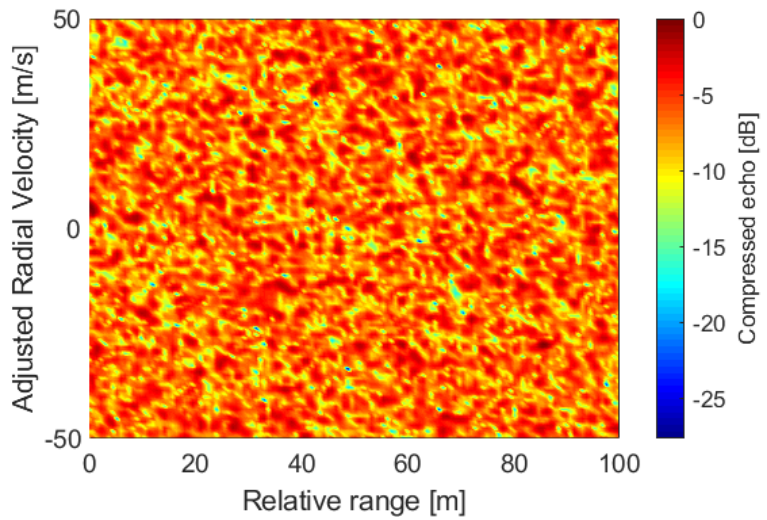

(a)

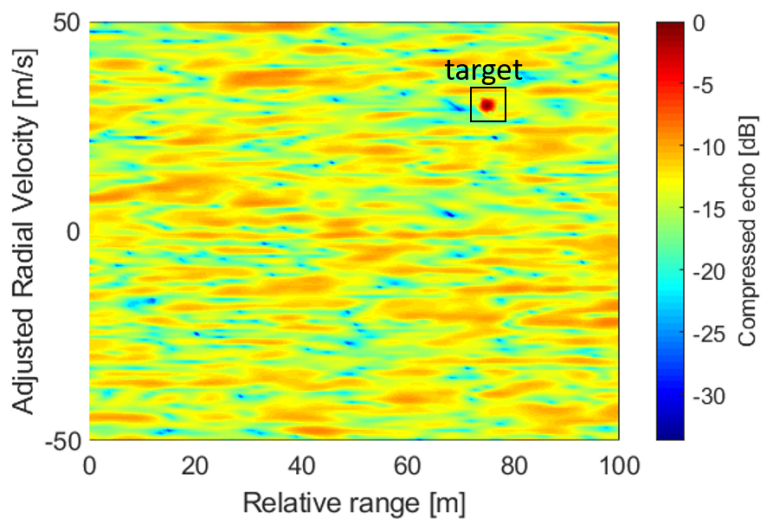

(b)

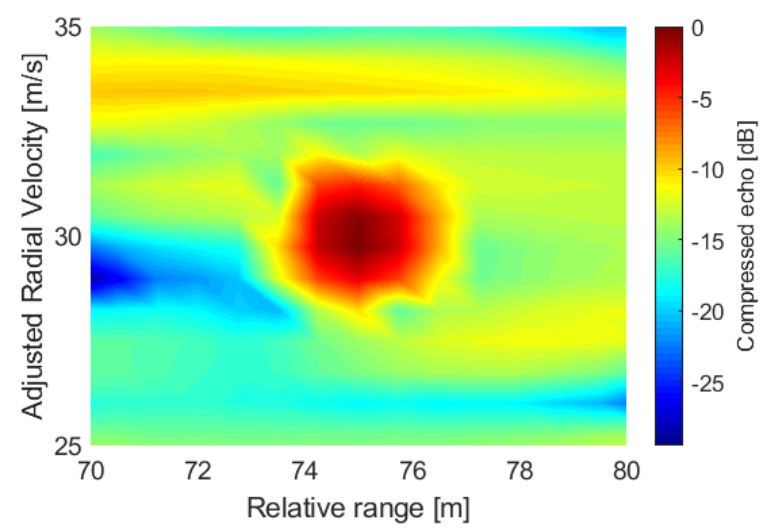

(c)

Fig. 6. Response pattern of signal detection vehicle equipped with an OFDM radar. In (a) the interference symbols occupy the whole channel of the signal of interest with $N=1024$ carriers and $M=512$ symbols. In (b) the interference overlaps with only 104 carriers for all symbols. (c) shows a zoomed-in plot of the response pattern for the area corresponding to the target of interest. which were configured to achieve the same resolution and an equivalent performance. We evaluated the effect of windowing and compared the signal of the target after processing and observed that the difference between the nature of the waveforms highly influences the sidelobe suppression in target detection. The Chebyshev window shows the best performance in all cases for both waveforms. Secondly, we did an interference simulation, where the interference is located at the same distance as the target in order to compare the one-way vs. two way signal attenuation. For the case of FMCW, this scenario allows us to detect the interference signal in a separate location with respect to the target. In a coherent case, the "target" due to interference could lead to the receiver saturation, while an incoherent interference can decrease the dynamic range of the signal. In the case of OFDM, the interference destroys the whole detection. Without implementing any interference cancellation, it may be possible to detect the signal of interest if the OFDM interferer carriers have a maximum overlapping of about $10 \%$ with the OFDM signal bandwidth. Orthogonal subcarrier allocations are one way to mitigate OFDM interference. Our study did not consider the cross-interference between FMCW and OFDM radars. A relevant work towards this was conducted in [24], where an algorithm involving cognitive radar [25] was used to suppress interference signal from FMCW radars in an OFDM radar system. For future studies, we plan to perform a comparative quantitative analysis of interference in FMCW and OFDM radars.

\section{ACKNOWLEDGMENT}

This research was supported by Vinnova grant 2018-01929.

\section{REFERENCES}

[1] C. Aydogdu, M. F. Keskin, G. Carvajal, O. Eriksson, H. Hellsten, H. Herbertsson, E. Nilsson, M. Rydström, K. Vanäs, and H. Wymeersch, "Radar interference mitigation for automated driving," IEEE Signal Processing Magazine, accepted for publication, 2020.

[2] W. Buller, B. Wilson, J. Garbarino, J. Kelly, B. J. Thelen, and B. M. Belzowski, "Radar congestion study," in No. DOT HS 812 632. United States. Department of Transportation. National Highway Traffic Safety Administration, 2018.

[3] M. Goppelt, H. . Blöcher, and W. Menzel, "Analytical investigation of mutual interference between automotive fmcw radar sensors," in 2011 German Microwave Conference, March 2011, pp. 1-4.

[4] Y. L. Sit, L. Reichardt, C. Sturm, and T. Zwick, "Extension of the OFDM joint radar-communication system for a multipath, multiuser scenario,' in 2011 IEEE RadarCon (RADAR), 2011, pp. 718-723.

[5] F. Liu, C. Masouros, A. Petropulu, H. Griffiths, and L. Hanzo, "Joint radar and communication design: Applications, state-of-the-art, and the road ahead," IEEE Transactions on Communications, Feb. 2020.

[6] B. Nuss, L. Sit, and T. Zwick, "A novel technique for interference mitigation in OFDM radar using compressed sensing," in 2017 IEEE MTT-S International Conference on Microwaves for Intelligent Mobility (ICMIM), March 2017, pp. 143-146.

[7] Y. L. Sit, C. Sturm, and T. Zwick, "Interference cancellation for dynamic range improvement in an OFDM joint radar and communication system," in 2011 8th European Radar Conference, Oct 2011, pp. 333-336.

[8] F. Uysal and S. Sanka, "Mitigation of automotive radar interference," in 2018 IEEE Radar Conference (RadarConf18), Oklahoma City, OK, USA, Apr. 2018, pp. 0405-0410. 
[9] M. Braun, R. Tanbourgi, and F. K. Jondral, "Co-channel interference limitations of OFDM communication-radar networks," EURASIP Journal on Wireless Communications and Networking, vol. 2013, no. 1, p. 207, Dec. 2013.

[10] S. Alland, W. Stark, M. Ali, and M. Hegde, "Interference in automotive radar systems: Characteristics, mitigation techniques, and current and future research," IEEE Signal Processing Magazine, vol. 36, no. 5, pp. 45-59, Sep. 2019.

[11] W. Wiesbeck and L. Sit, "Radar 2020: The future of radar systems," in 2014 International Radar Conference, Lille, France, Oct. 2014, pp. 1-6.

[12] M. Braun, "OFDM radar algorithms in mobile communication networks," Ph. D. dissertation, 2014.

[13] J. Fink and F. K. Jondral, "Comparison of OFDM radar and chirp sequence radar," in 2015 16th International Radar Symposium (IRS), June 2015, pp. 315-320.

[14] — "A numerical comparison of chirp sequence versus OFDM radar waveforms," in 2015 IEEE 82nd Vehicular Technology Conference (VTC2015-Fall), Sep. 2015, pp. 1-2.

[15] S. M. Patole, M. Torlak, D. Wang, and M. Ali, "Automotive radars: A review of signal processing techniques," IEEE Signal Processing Magazine, vol. 34, no. 2, pp. 22-35, 2017.

[16] M. A. Richards, Fundamentals of Radar Signal Processing. McGrawHill Education, 2005.

[17] C. Sturm, T. Zwick, and W. Wiesbeck, "An OFDM system concept for joint radar and communications operations," in VTC Spring 2009 - IEEE 69th Vehicular Technology Conference, April 2009, pp. 1-5.

[18] C. Sturm and W. Wiesbeck, "Waveform design and signal processing aspects for fusion of wireless communications and radar sensing," Proceedings of the IEEE, vol. 99, no. 7, pp. 1236-1259, July 2011.

[19] B. Schweizer, C. Knill, D. Schindler, and C. Waldschmidt, "Steppedcarrier OFDM-radar processing scheme to retrieve high-resolution range-velocity profile at low sampling rate," IEEE Transactions on Microwave Theory and Techniques, vol. 66, no. 3, pp. 1610-1618, March 2018.

[20] N. Dinur and D. Wulich, "Peak-to-average power ratio in high-order ofdm," IEEE Transactions on Communications, vol. 49, no. 6, pp. 1063 1072,2001

[21] R. G. Lyons, Understanding Digital Signal Processing (2nd Edition). USA: Prentice Hall PTR, 2004.

[22] A. Antoniou, Digital Signal Processing, 2nd ed. Mcgraw-Hill, 2016.

[23] G. M. Brooker, "Mutual interference of millimeter-wave radar systems," IEEE Transactions on Electromagnetic Compatibility, vol. 49, no. 1, pp. 170-181, Feb 2007.

[24] G. Hakobyan, "Orthogonal frequency division multiplexing multipleinput multiple-output automotive radar with novel signal processing algorithms," Ph. D. dissertation, 2018.

[25] S. Haykin, "Cognitive radar: a way of the future," IEEE Signal Processing Magazine, vol. 23, no. 1, pp. 30-40, 2006. 